\title{
Left atrial involvement of a right lung tumour
}

\author{
Phuong Bui, MD • Oscar D. Aljure, MD • Nestor Villamizar, MD • Dao M. Nguyen, MD • \\ Yiliam F. Rodriguez-Blanco, MD
}

Received: 28 October 2015/Revised: 17 November 2015/Accepted: 1 December 2015/Published online: 10 December 2015

(c) Canadian Anesthesiologists' Society 2015

A 27-yr-old man with a history of a non-seminomatous germ cell tumour and orchiectomy with adjuvant chemotherapy presented to our institution with a giant right lung tumour that extended through the right superior pulmonary vein (RSPV) into the left ventricle (Figure 1A). Biopsy of the lung mass revealed a metastatic germ cell tumour, and the patient underwent urgent chemotherapy with excellent response. A residual $5.3 \mathrm{~cm} \times 4.9 \mathrm{~cm} \times 4.9$ $\mathrm{cm}$ tumour remained centred in the right middle lobe with extension from the RSPV into the left atrium (LA) (Figure 1B). Once maximal cytoreduction and normalization of tumour markers were achieved, the patient was brought to the operating room for right upper and middle lobe bilobectomy as well as resection of the LA tumour. Intraoperative two-dimensional (2D) and threedimensional (3D) transesophageal echocardiography (TEE) showed a $1.6 \mathrm{~cm} \times 1.8 \mathrm{~cm}$ non-mobile mass in the LA (Figure 1C). The surgeon attempted retraction and manipulation of the tumour; however, TEE showed that the mass remained immobile and too large for excision from the pulmonary vein side without opening the LA. Therefore, cardiopulmonary bypass was initiated, and the patient underwent resection of the intra-atrial portion of the tumour and removal of the entire mass en bloc with the right upper and middle lobe.

A variety of imaging modalities are available to aid in the characterization of intracardiac masses and in planning surgical resection. Transesophageal echocardiography is the intraoperative modality of choice, with both 2D and 3D TEE providing excellent information on tumour size, location, morphology, and any attachment sites. These modalities also allow for dynamic assessment of the consequences of intracardiac masses on heart function and hemodynamics. This case stresses the vital role of intraoperative TEE as an indispensable tool for managing intracardiac masses and for guiding surgical decision making.

P. Bui, MD $(\bowtie) \cdot$ O. D. Aljure, MD .

Y. F. Rodriguez-Blanco, MD

Department of Anesthesiology, Perioperative Medicine and Pain Management, University of Miami, Miller School of Medicine, Miami, FL, USA

e-mail: phuonglan.bui@jhsmiami.org

N. Villamizar, MD · D. M. Nguyen, MD

Department of Surgery, University of Miami, Miller School of Medicine, Miami, FL, USA 

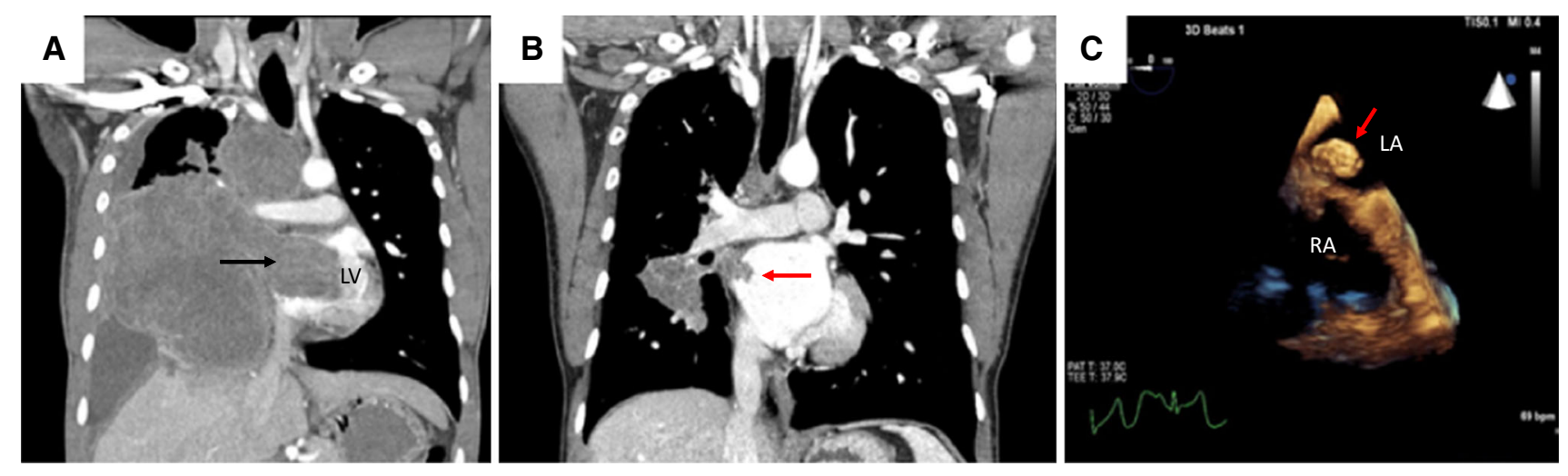

Figure 1 A) Computerized tomography (CT) of the chest before chemotherapy showing a large infiltrative mass (black arrow) occupying two-thirds of the right hemithorax with direct extension into the left atrium (LA) and left ventricle (LV). There is partial occlusion of the mitral valve opening and the left ventricular outflow tract. B) After chemotherapy, the chest CT shows a markedly reduced

mass centred in the right middle lobe, but with continued extension into the LA (red arrow) at its confluence with the right superior pulmonary vein. C) A mid-esophageal three-dimensional transesophageal echocardiography image depicting the pre-resection LA mass (red arrow)

Conflicts of interest None. 